\title{
The Recursive Jigsaw Reconstruction Technique
}

\section{Paul Jackson*}

University of Adelaide

E-mail: p.jackson@adelaide.edu.au

\begin{abstract}
The observation of light super-partners from a supersymmetric extension to the Standard Model is an intensely sought-after experimental outcome, providing an explanation for the stabilization of the electroweak scale and indicating the existence of new particles which could be consistent with dark matter phenomenology. We introduce a new technique called the Recursive Jigsaw Reconstruction, a method for decomposing, event-by-event, measured quantities into a basis of complementary observables that can be used to develop more sensitive measurements and searches in collider experiments. In searches for supersymmetry where pair-produced sparticles decay into standard model particles and lightest supersymmetric partners, manifested as large missing transverse momentum, these observables provide sensitivity to scales in the event, evaluate visible decay products in estimations of their production frame and encode information about the particle properties (spin, $\mathrm{CP}$ etc) in angular variables. Herein, we introduce the technique, and discuss applying it to extract a collection of kinematic observables which can be used to probe compressed scenarios, in particular exploiting the correlation between missing momentum and that of radiative jets.
\end{abstract}

38th International Conference on High Energy Physics 3-10 August 2016

Chicago, USA

\footnotetext{
${ }^{*}$ Speaker.
} 


\section{Introduction}

The general purpose LHC discovery experiments, ATLAS and CMS, were built with two goals in mind: the discovery and measurement of the Higgs boson and to search for evidence for beyond standard model (BSM) physics at a new scale. The first of these was achieved during Run 1 and the second is now the key challenge of the LHC physics program. Such BSM physics processes may be just out of reach of the available dataset, or may be manifested in difficult-to-measure channels. In the spirit of covering every corner of phase space, it behooves us to consider all possibilities in the techniques we employ to search for BSM physics, as perhaps further improvements can be made. With this in mind we introduce the Recursive Jigsaw Reconstruction (RJR) technique which can be used to investigate BSM theories characterized by a discrete $\mathbb{Z}_{2}$ symmetry in collider experiments. The RJR method approximates recursively, event-by-event, the relevant frames of reference and introduces a new basis of kinematic and angular Jigsaw variables. Final states from R-parity conserving supersymmetry (SUSY) models are characterised by an even number of lightest supersymmetric particles (LSP), likely two, undetected by the detector and contributing to the missing transverse momentum, $\vec{H}_{T}$. By applying the RJR technique, through a series of "Jigsaw Rules" we can contextualize the $\vec{B}_{T}$, partitioning it between two decay hemispheres and extract a basis of complementary variables. These variables replace conventional, detector derived, laboratory frame variables with a more physics-driven basis that can be used to characterize events and provide, where possible, uncorrelated observables to isolate signal-like events from backgroundlike events. In compressed scenarios, the same principles can be applied to a different view of the events yielding a more relevant basis of observables.

\section{Recursive Jigsaw Reconstruction}
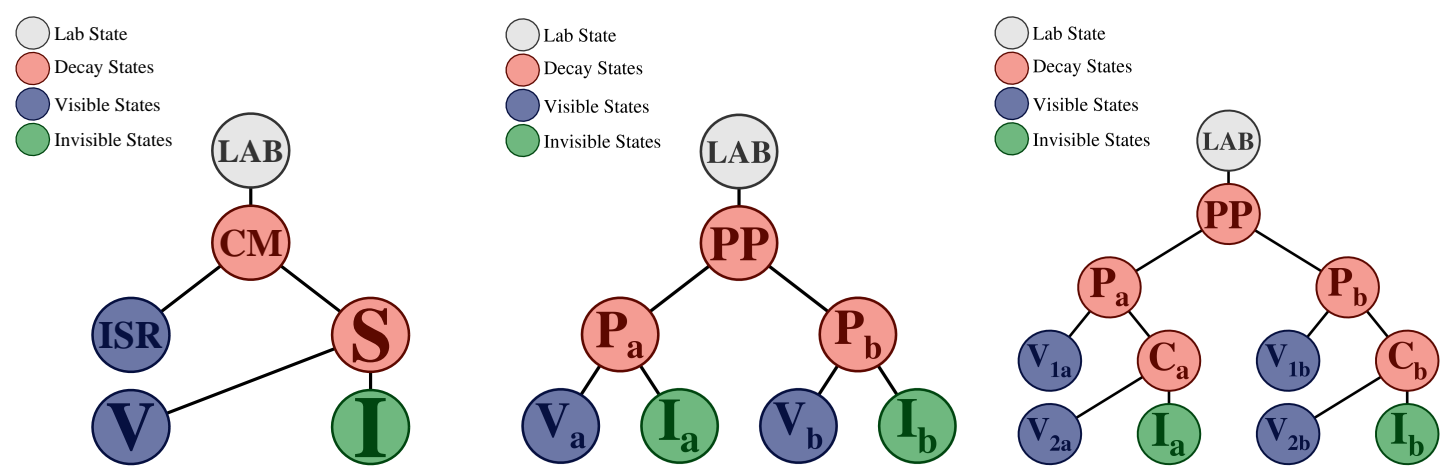

Figure 1: Decay tree diagrams for application to compressed spectra (left), pair produced parent particles " $\mathrm{P}$ " decaying to visible and invisible final states (center) and pair produced particles decaying through intermediate children systems " $\mathrm{C}$ " to sets of visible and invisible final state particles (right).

RJR is a new approach to reconstructing open final states. The strategy is to transform observable momenta iteratively reference-frame to reference-frame, traveling through each of the reference frames relevant to the topology. At each step we extremize only the relevant degrees of freedom related to that transformation. We repeat the procedure recursively according to particular 
rules defined for each topology (the topology relevant to each reference frame). In Figure 1 we show three decay tree diagrams for (left) compressed searches, where the mass between the parent particle and lightest daughter particle is small, and for pair-produced systems decaying either directly (center), or through an intermediate child (right), to pairs of invisible particles.

\section{Application to compressed spectra}

In [3] a set of observables are introduced that provide increased sensitivity to studying compressed scenarios at collider experiments. They are designed for the analysis of events where one or more strong initial state radiation (ISR) jets are present, causing the system of initially produced sparticles to recoil in the opposite direction. In the limit where the LSPs receive no momentum from their parents' decays, the $\vec{H}_{T}$ results solely from the recoil against ISR, and the following approximation holds:

$$
\vec{E}_{T} \sim-\vec{p}_{T}^{\mathrm{ISR}} \times \frac{m_{\tilde{\chi}}}{m_{\tilde{P}}},
$$

where $\vec{p}_{T}^{\text {ISR }}$ is the total ISR system transverse momentum.

In the decay tree on the left in Figure 1, we can assigned each reconstructed object hypothesized to come from the decay of sparticles in the event to the " $V$ " system, while those identified as initial state radiation are associated with "ISR". With the missing momentum reconstructed in each event interpreted as the system "I", the total sparticle system ("S") and center-of-mass system of the whole reaction ("CM") are defined as the sum of their constituents. With the four-vectors of each element of the decay tree specified, an estimator of the quantity $\left|\vec{H}_{T}\right| /\left|\vec{p}_{T}^{\mathrm{ISR}}\right|, R_{\mathrm{ISR}}$, is calculated as:

$$
R_{\mathrm{ISR}} \equiv \frac{\left|\vec{p}_{\mathbf{I}, T}^{\mathbf{C M}} \cdot \hat{p}_{\mathbf{I S R}, T}^{\mathbf{C M}}\right|}{\left|\vec{p}_{\mathbf{I S R}, T}^{\mathbf{C M}}\right|},
$$

where subscripts indicate the system and superscripts the reference frame the momentum is evaluated in. Using these key variables, and others derived from the kinematic basis described in [3] it was demonstrated that gluinos(squarks) up to mass of $1400(900) \mathrm{GeV}$, with a mass difference between parents and LSP of up to $200 \mathrm{GeV}$ can be excluded with $100 \mathrm{fb}^{-1}$ of data.

\section{Other applications}

The general decay tree diagrams in Figure 1 can be applied to a variety of more complicated topologies, on the left of Figure 2 we consider di-leptonic top. In this case the two hemispheres can be largely decoupled, and the decay chains can be reconstructed independently thus leading to a two dimensional mass peak reconstructed at the anticipated scale of the process. One can imagine that such scale variables, in concert with additional handles would be a powerful tool to search for the SUSY analogue of the top quark. For other open final states such as $H \rightarrow W^{+} W^{-} \rightarrow \ell^{+} v \ell^{-} \bar{v}$, depicted to the right of Figure 2 we can resolve a mass peak situated at the Higgs mass. Angular variables relating the boosts between reference frames encode information about the particles in the decay tree (i.e. spin, CP etc), and can be used not only to suppress backgrounds from the standard model that have different properties but also provide sensitivity to the signal characteristics themselves. 
Beyond these two examples, any decay tree can be drawn from a set of visible and invisible states, with intermediate decay states, and the rules to assess quantities in these frames can be applied. In general this will yield variables sensitive to mass differences between decay states, decay angles of visible objects produced in their rest frames and azimuthal angles between these frames. This has been demonstrated to work, and utilized, in searches for pair-production of squarks and gluinos decaying to jets and $\vec{H}_{T}$, applied to production of $\chi_{2}^{0} \chi_{1}^{ \pm}$and production of pairs of third generation squarks $(\tilde{t})$ decaying to produce $b$-jets, jets, leptons and $\overrightarrow{\mathscr{H}}_{T}$. These results will be summarized in the literature [1] and are contained in a code framework making it possible for any user to reproduce them [2].
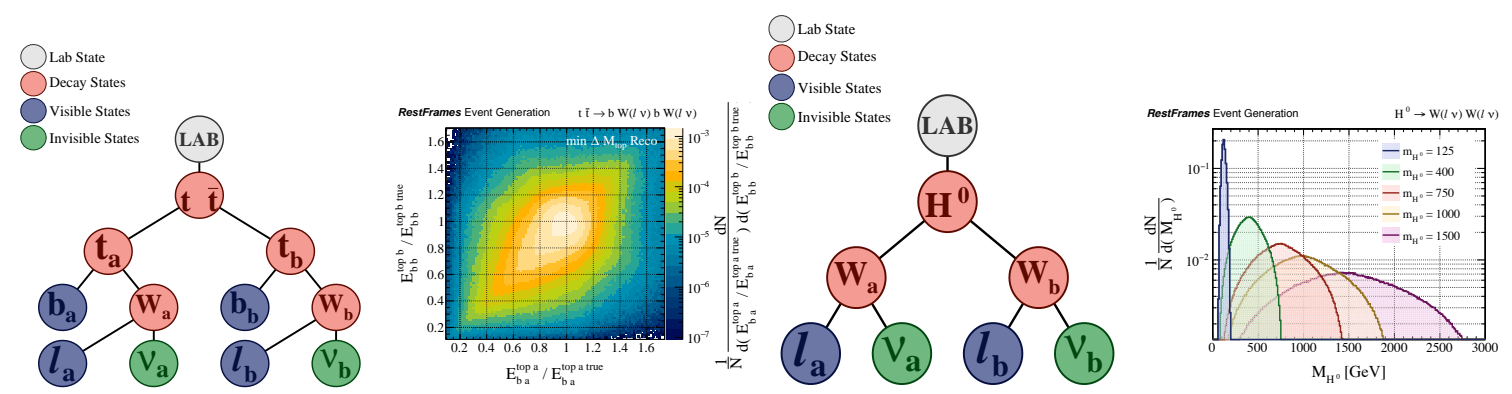

Figure 2: The decay tree diagram for dileptonic $t \bar{t}$ decays and the scale sensitive variable for the two top decay hemispheres, normalised by their true values (left). The decay tree diagram for $H \rightarrow W W$ and the reconstructed resonance mass for different generated resonance masses, all measured at their true value (right).

\section{Summary}

The RJR provides a technique to decompose events into a complete, well-defined and largely uncorrelated basis of variables. The technique aims to remove redundancy and, as much as possible, "diagonalize" the available information to match observables with the particle masses and decay angles we hope to discover/measure. The technique has already been applied successfully to searches at the LHC for compressed SUSY in top squark decays [4] and jets $+\vec{H}_{T}$ [5]. The RJR improves upon conventional analysis methods, while being complementary to them, and provides an exciting new way to search for evidence of BSM physics.

\section{References}

[1] P. Jackson, C. Rogan, The Recursive Jigsaw Reconstruction - In preparation.

[2] C. Rogan, RestFrames, http://RestFrames.com

[3] P. Jackson, C. Rogan and M. Santoni, Sparticles in Motion - getting to the line in compressed scenarios with the Recursive Jigsaw Reconstruction arXiv:1607.08307.

[4] ATLAS Collaboration, "Search for the Supersymmetric Partner of the Top Quark in the Jets $+\vec{Z}_{T}$ Final State at $\sqrt{s}=13 \mathrm{TeV}$ " ATLAS-CONF-2016-077

[5] ATLAS Collaboration, "Further searches for squarks and gluinos in final states with jets and missing transverse momentum at $\sqrt{s}=13 \mathrm{TeV}$ with the ATLAS detector" ATLAS-CONF-2016-078 